\title{
WHITE DWARF COOLING CURVES AND SEARCHES FOR WHITE DWARFS
}

\author{
MATT A. WOOD \\ Florida Institute of Technology \\ Department of Physics and Space Sciences \\ Melbourne, FL, 32901-6988, USA \\ wood@kepler.pss.fit.edu
}

\section{White Dwarf Cooling Curves}

White dwarf (WD) evolution is essentially a cooling problem in two parts. Degenerate electrons in the core provide the support while non-degenerate $\mathrm{C}$ and $\mathrm{O}$ ions provide the thermal reservoir (Koester \& Chanmugam 1990, Kawaler 1996). The rate of energy loss through the non-degenerate envelope of $\mathrm{H}$ and $\mathrm{He}$ - segregated by gravitational settling - is meager enough that the vast majority of WDs have not had time to cool to undetectability in the $\sim 10$ Gyr age of the Galactic disk. Because WDs are a remarkably homogeneous class with a mass function sharply peaked about $0.6 M_{\odot}$, and because the cooling physics is relatively straightforward to calculate, the observed deficit of WDs below $M_{V}=16.5\left[\log \left(L / L_{\odot}\right) \approx-4.5, T_{\text {eff }} \approx 4000 \mathrm{~K}\right.$; see Liebert et al. 1988 (LDM), Oswalt et al. 1996 (OSWH), and Leggett et al. 1997] provides constraints on the age and starformation history of the Galactic disk (Winget et al. 1987 (WEA97), Wood 1992 (W92), Yuan 1992, Hernanz et al. 1994).

The maximum fractional surface layer masses that can remain following post-AGB evolution are $\log \left(q_{\mathrm{H}}\right) \sim-4$ and $\log \left(q_{\mathrm{He}}\right) \sim-2$ (for a $0.6 M_{\odot}$ remnant-the values are inversely correlated with remnant mass), although we know from WD asteroseismology that surface $H$ layer masses range from this value to smaller than $\log q_{\mathrm{H}} \sim-6$ (e.g., Fontaine \& Wesemael 1997). Some $75 \%$ of WDs in the McCook \& Sion (1987) catalog reveal pure-H photospheres (spectral type DA). The remainder are broadly classified as non-DAs, with most showing show pure-He spectra (DB) or continuous spectra (DC), and with a handful that show mixed compositions (DAB), or C (DQ) or metal lines (DZ). See Bergeron et al. 1997 for a thorough discusion of the chemical evolution of cool WDs.

As a hot WD, cooling is dominated by neutrino processes which can "out shine" the photon luminosity by $\lesssim 100$ near $T_{\text {eff }} \sim 10^{5} \mathrm{~K}\left(\log \left(L / L_{\odot}\right) \sim 2\right.$, again for a $0.6 M_{\odot}$ model), which results in a core temperature inversion, and which accelerates the cooling (Fig. 1). Neutrinos become unimportant below $\log \left(L / L_{\odot}\right) \sim-1.6\left(T_{\text {eff }} \sim 20,000 \mathrm{~K}\right)$; at this point, the models are closest to the Mestel (1952) ideal, with nearly isothermal cores and radiative envelopes. A significant surface convection zone develops near $T_{\text {eff }} \sim 10^{4} \mathrm{~K}$ which persists from there on, reaching a maximum fractional depth in mass of $\log \left(q_{\mathrm{cz}}\right) \approx-6$ and potentially mixing with the subsurface He layer. Cool WDs $\left(T_{\text {eff }} \lesssim 7000 \mathrm{~K}, \log \left(L / L_{\odot}\right) \lesssim-3.5\right)$ undergo a crystallization phase transition of their $\mathrm{C}-\mathrm{O}$ interiors. The release of latent heat associated with this first-order phase transition slows the cooling temporarily, but quantum effects in this phase act to lower the heat capacity and accelerate cooling. Based on the spindle form of the phase diagram of the C-O binary ionic mixture (e.g., Segretain et al. 1994, Isern et al. 1997), Hernanz et al. (1994 and references therein), have suggested that the solidification phase transition should be accompanied by a global redistribution that effectively moves $\mathrm{O}$ inward and $\mathrm{C}$ outward, and that acts as an additional energy source comparable in magnitude to the latent heat released. Detailed evolutionary models incorporating this process have recently been published by Salaris et al. (1997). The models shown in Fig. 1 include the latent heat release but phase separation is not implemented. The age-luminosity relation must be extrapolated below $\log \left(L / L_{\odot}\right) \sim-5.5$. 

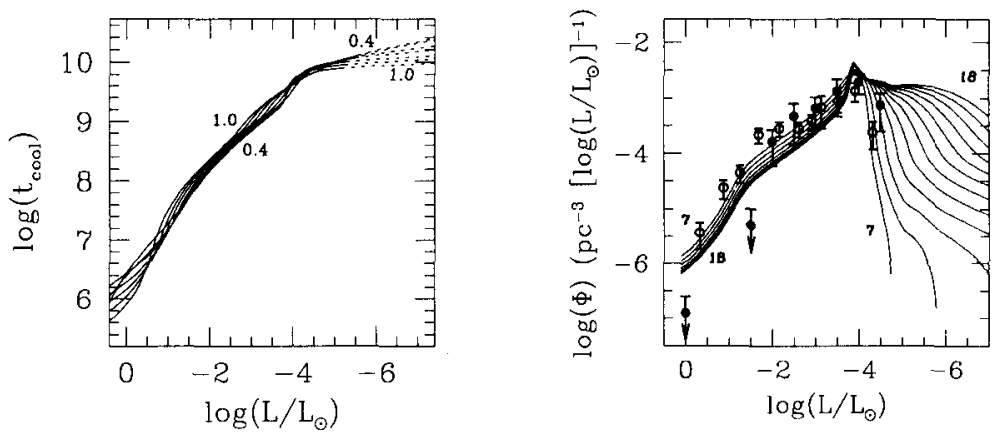

Figure 1. Left panel shows cooling curves of C-O core DA models with masses ranging from 0.4 to $1.0 M_{\ominus}$, as indicated. Extrapolations are shown as dashed lines. Right panel shows integrated WD LFs from Wood (1995) shown over the data of LDM (open circles) and OSWH (filled circles). Ages range from 7 to 18 Gyr, as indicated.

\section{Searches for White Dwarfs}

Most hot WDs have been found through colorimetric surveys searching for faint blue objects [Green et al. 1986 (PG); Kondo et al. 1984 (Kiso); Demers et al. (1986) Lamontagne et al. 1997 (MCT); Stobie et al. 1997 (EC); Cristiani et al. 1995 (HBQS)]. Cool WDs are faint and therefore must be close if they are to be detected. Their photometric colors are similar to $\mathrm{K}$ dwarfs and therefore the majority of cool WDs have been found through proper motion surveys (e.g., Luyten 1978, 1979 (etc.); Giclas et al. 1971, 1989; Ruiz 1996 and references therein) with extensive spectroscopic followup work (e.g. Oswalt et al. 1991, 1993). Chuck Claver and collaborators (1998, in preparation) are using a $\mathrm{MgH}$ and $\mathrm{CaH}$ filter to separate WD candidates from the lower MS in a photometric CCD survey; spectroscopic followup observations are being made using WIYN Hydra. Recently, WDs have been detected in open and globular clusters with HST (Richer et al. 1995, 1997; Cool et al. 1996; Renzini et al. 1996).

\section{White Dwarfs and the Age of the Galactic Disk}

Much of the recent excitement surrounding WDs centers on their use as age indicators for the Galactic disk (WEA87, W92, OSWH), and the MACHO collaboration result indicating that up to $50 \%$ of the mass of the halo may be composed of WDs (Alcock et al. 1993, 1997). Using a sample of common proper motion binary WDs and updated evolutionary models (Wood 1995), OSWH find a most likely age of $\sim 9.5 \mathrm{Gyr}$, where the data of LDM fit with the same models suggest an age $\sim 2$ Gyr younger (see Fig. 1). Monte Carlo simulations (Wood \& Oswalt 1998) suggest that sample-to-sample variations are large, and that inferred ages should be considered lower limits. The 10 Gyr WD ages compare favorably with the 8-12 Gyr Hubble expansion ages and the recent 12 Gyr globular cluster age estimates (e.g., Chaboyer et al. 1997).

Alcock et al. $(1993,1997)$ report a significant population of $\sim 0.5 M_{\odot}$ WDs in the galactic halo. In order for this population to exist in kinematically significant numbers and not have been detected via other means, it must have been the result of an enormous star formation burst $\sim 15$ Gyr ago with an IMF sharply peaked near $M_{\mathrm{MS}} \sim 3 M_{\odot}$ (Tamanaha et al. 1990, Ryu et al. 1990, Adams \& Laughlin 1996, Chabrier et al. 1996). Adopting such an IMF, Fields et al. (1997) modeled schematically the formation of baryonic galactic halos by following the dynamics, chemical evolution, etc. on a hierarchy of mass scales, finding a best-fit model that yielded halos that were $40 \%$ baryonic and dominated by WDs. However, Fields et al. only considered the global metallicity $Z$. Gibson \& Mould (1997) revisited the problem following the individual elemental abundances and found that all such models overproduce $\mathrm{C}$ and $\mathrm{N}$ relative to $\mathrm{O}$ by a factor of 10 or more. In addition, Kawaler (1996) notes that up to 12 faint halo WDs should be visible in the Hubble Deep Firld, but. Flym et al. (1996) found no objects with $V-I>1.8$. To date, we must conclude that no completely satisfying explanation of the origin of the MACHO collaboration objects exists. 


\section{References}

Adams, F.C. \& Laughlin G. 1996, ApJ, 468, 586

Alcock, C., et al. 1993, Nature, 365, 621

Alcock, C., et al. 1997, ApJ 486, 697

Bergeron, P., Ruiz, M.-T., and Leggett, S.K. 1997, ApJS, 108, 339

Chaboyer, B., Demarque, P., Kernan, P.J., \& Krauss, L.M. 1997, ApJ, in press.

Chabrier, G., Segretain, L. \& Méra, D. 1996, ApJ, 468, L21

Cristiani, S., et al. 1995, A\&A Suppl. Ser., 112, 347

Cool, A.M., Piotto, G., \& King, I.R. 1996, ApJ, 468, 655

Demers, S., Kibblewhite, E.J., Irwin, J.J., Nithakorn, D.S., Béland, S., Fontaine, G., \& Wesemael, F. 1986, AJ, 92, 878

Fields, B.D., Mathews, G.J. \& Schramm, D.N. 1997, ApJ, 483, 625

Flynn, C., Gould, A., \& Bahcall, J.N. 1996, ApJL, 466, L55

Fontaine, G. \& Wesemael, F. 1997, in White Dwarfs, eds. I. Isern et al. (Kluwer: Dordrecht) p. 173

Giclas, H.L., Burnham, R., Thomas, N.G. Lowell Proper Motion Survey, Northern Hemisphere Catalog (Flagstaff: Lowell) (1971).

Giclas, H., Burnham, R., Thomas, G. Lowell Proper Motion Survey, Southern Hemisphere Catalog. Lowell Obs. Bull. 164 , Vol. VIII, p.89 (1978).

Gibson, B.K. \& Mould, J.R. 1997, ApJ, 482, 98

Green, R. G., Schmidt, M., \& Liebert., J. 1986, ApJS, 61, 305

Hernanz, M., García-Berro, E., Isern, J., Mochkovitch, R., Segretain, L., \& Chabrier, G. 1994, ApJ, 434, 652

Isern, J. Mochkovitch, R. García-Berro, E. \& Hernanz, M. 1997, ApJ, 485, 308

Kawaler, S.D., Novikov, I., \& Srinivasan, G. 1997, Saas-Fee Adv. Course 25 (New York: Springer) eds. G. Meynet \& D. Schaerer

Kawaler, S.D. 1996, ApJL, 467, L61

Koester, D., \& Chanmugam, G. 1990, Rep. Prog. Physics, 53, 837

Kondo, M., Maehara, H., \& Noguchi, T., 1984, Ann. Tokyo Astron. Obs., 20, 130

Lamontagne, R., Wesemael, F., Fontaine, G., Demers, S., Bergeron, P., Irwin, M.J., and Kunkel, W.E. 1997, in White Dwarfs, eds. I. Isern et al. (Kluwer: Dordrecht) p. 143

Leggett, S.K., Ruiz, M.-T., \& Bergeron, P., 1998, ApJ, in press.

Liebert, J., Dahn, C. C., \& Monet, D. G. 1988, ApJ, 332, 891

Luyten, W.J. 1978, Proper Motion Survey with the 48-inch Schmidt Telescope: L Double Stars with Common Proper Motion (Minneapolis: University of Minnesota)

Luyten, W. J. 1979, The LHS Catalogue, Second Edition (Minneapolis: University of Minnesota)

McCook, G.P., \& Sion, E.M. 1987, ApJS, 65, 603

Mestel, L. 1952, MNRAS, 112, 583

Oswalt, T.D., Sion, E.M., Hintzen, P.M. \& Liebert, J. 1991, in White Dwarfs, eds. G. Vauclair \& E.M. Sion (Kluwer: Dordrecht), p. 379

Oswalt, T.D. et al. 1993, in White Dwarfs: Advances in Observation \& Theory, ed. M. Barstow (Kluwer: Dordrecht) p. 419

Oswalt, T. D., Smith, J. A., Wood, M. A., \& Hintzen, P. M., 1996, Nature, 382, 692

Renzini, A., et al. 1996, ApJ, 465, L23

Richer, H.B. et al. 1995, ApJ, 451, L17

Richer, H.B., et al. 1997, ApJ, 484, 741

Ruiz, M.-T. 1996, AJ, 111, 1267

Ryu, D., Olive, K. A., \& Silk, J. 1990, ApJ, 353, 81

Salaris, M., Dominguez, I., García-Berro, E., Hernanz, M., Isern, J., \& Mochkovitch, R. 1997, ApJ, 486, 413

Sion, E.M. 1986, PASP, 98, 821

Segretain, L., Chabrier, G., Hernanz, M., García-Berro, E., Isern, J., \& Mochkovitch, R. 1994, ApJ, 434, 641

Stobie, R.S., et al. 1997, MNRAS, 287, 848

Tamanaha, C.M., Silk, J., Wood, M.A., and Winget, D.E. 1990 ApJ, 358, 164.

Winget, D. E., Hansen, C. J., Liebert, J., Van Horn, H. M., Fontaine, G., Nather, R. E., Kepler, S. O., \& Lamb, D. Q. 1987, ApJ, 315, L77

Wood, M. A. 1992, ApJ, 386, 539

Wood, M.A. 1995, in White Dwarfs, eds. D. Koester and K. Werner (Berlin: Springer-Verlag), p. 41

Wood, M.A. \& Oswalt T.D. 1998, ApJ, in press.

Yuan, J. W. 1992, A\&A, 261, 105 